\title{
Correlations between Rheological Behavior and Intrinsic Properties of Nanofibrillated Cellulose from Wood and Soybean Hulls with Varying Lignin Content
}

\begin{abstract}
Maria C. Iglesias, ${ }^{\text {a }}$ Fatima Hamade, ${ }^{\mathrm{b}}$ Burak Aksoy, ${ }^{\mathrm{b}, \mathrm{c}}$ Zhihua Jiang, ${ }^{\mathrm{b}, \mathrm{c}}$ Virginia A. Davis, and Maria S. Peresin ${ }^{\mathrm{a}, *}$

Effects of raw material and chemical composition were considered relative to the intrinsic properties and the rheological behavior of nanofibrillated cellulose aqueous suspensions (CNFs). Atomic force microscopy, Fouriertransform infrared spectroscopy, surface chemistry analysis, thermal gravimetrical analysis, and zeta-potential were used to study the morphology, chemical composition, charge density, as well as thermal and colloidal stability of the different CNFs. Regarding the rheological properties of the samples, steady-state and oscillation studies of the CNF aqueous suspensions obtained from wood and soybean hulls were performed. An interesting correlation was found between the rheological behavior of CNF suspensions and their intrinsic properties. Soybean CNF presented lower viscosities than wood samples, which could be related to their morphology and charge density. Additionally, unbleached soybean CNF (sb-LCNF) showed yield stress compared with the other samples, which could be attributed to the presence of pectin. Furthermore, the different chemical compositions between the samples affected their thermal properties, as well as on their crystallinity.
\end{abstract}

Keywords: Lignin-containing cellulose nanofibrils; LCNFs; Rheology; Residual lignin; Ligno-nanofibers; Cellulosic nanofibers; Soybean hulls; Soybean cellulose nanofibrils

Contact information: a: Forest Products Development Center, School of Forestry and Wildlife Sciences, Auburn University, 520 Devall Dr. AL 36849 USA; b: Department of Chemical Engineering, Samuel Ginn College of Engineering, Auburn University, 345 West Magnolia Ave, AL 36849 USA; c: Alabama Center for Paper and Bioresource Engineering, 356 Ross Hall, AL 36849, USA.

*Corresponding author: soledad.peresin@auburn.edu

\section{INTRODUCTION}

Over the last decade, the utilization of nanocellulose for novel applications, such as stabilizers for Pickering emulsions, 3D printing, carriers for drug delivery, and packaging have positioned this renewable source as a promising alternative substitution for petroleum-based materials (Hubbe et al. 2017a). Nanocellulose is well known for being obtained through the isolation of cellulose fibers and transformed to the nanoscale by chemical and/or mechanical treatments. Furthermore, nanocellulose has shown remarkable properties such as the aspect ratio, low density, and its ability to be chemically modified (Klemm et al. 2011).

Although commercial nanocellulose is mainly isolated from wood sources, over the years different resources have been found as alternatives for nanocellulose production. This opens the possibility of conferring value to side streams of agroindustry that were often considered underutilized waste streams. Banana (Tarrés et al. 2017) and pineapple leaves (Deepa et al. 2015), sugarcane bagasse (Feng et al. 2018), lotus leaf stalks (Chen et al. 
2015), jute (Ahuja et al. 2018), cotton (Sangeetha et al. 2019), corn stover (Xu et al. 2018), waste from the agave plant (Palacios Hinestroza et al. 2019) and pomelo peel (Tang et al. 2020b), are some of the alternative raw materials presented on the literature.

Soybean is one of the most relevant agricultural sources in the United States (US). Within its composition, soybean contains proteins, oils, carbohydrates, and ash in the amount of 40, 21, 34, and 4\%, respectively (Kawamura 1967). In 2019, the US was reported as the second leading worldwide soybean producer with $27 \%$ of the total production (FAOSTAT 2019). During the same year, $64.7 \%$ of this production was crushed to extract the oil contained in the seed (USDA 2019), giving little commercial value to the remaining fibrous material named soybean hulls. This by-product is also known as seed coat and is mainly utilized as a fiber source for cattle (Hult et al. 2010).

Due to their availability and their chemical composition versatility, several researchers have focused on utilizing soybean fibers as a source for nanocellulose production. Efforts to extract fibrous materials from soybean hulls has also been driven by efforts to increase the value of this waste material (Debiagi et al. 2020).

Debiagi et al. 2020 obtained nanofibrillated cellulose, which is also known as cellulose nanofibril (CNF) by reactive extrusion followed by bleaching of the soybean hulls and studied how this process affected the properties of the nanofibers with respect to the original soybean fibers. Additionally, Li et al. (2019) studied the effect of alkaline and acidic pretreatments on the fiber properties, corroborating how their properties can be altered depending on the extraction/purification methods. Similarly, Ferrer et al. (2016) characterized microfibers and microparticles obtained from soybean utilizing mechanical and chemical processes and compared their properties with CNF obtained from fully bleached cellulose pulp. Due to their abundance and versatility, soybean hull-based CNFs have recently been utilized in the development of novel applications such as in feed binders and in gas detectors for food spoilage and ripening (Aksoy et al. 2020).

This work focused on comparing the effect of the chemical composition of soybean hulls and wood fibers on the rheological behavior of bleached and unbleached nanocellulose suspensions. Rheology provides a useful assessment of differences between materials, since properties vary with concentration, material structure, and interactions that are affected by surface chemistry. In addition, the materials were characterized by atomic force microscopy (AFM) to determine structure and dimensions, X-ray diffraction (XRD) for crystal structure, Fourier-transform infrared spectroscopy (FTIR) for chemical composition, surface charge for charge density, and thermogravimetric analysis (TGA) for thermal stability. These techniques provide insight into the materials' morphology and resulting microstructure.

\section{EXPERIMENTAL}

\section{Materials}

Never-dried hardwood kraft pulps: i) bleached (lignin content of $<0.1 \%$ ), and ii) unbleached (lignin content of $2.25 \%$ ) were kindly provided by a US mill. Soybean hulls were provided by Republic Mills, Inc., of Okolona, Ohio. Fibers from the soybean hulls were extracted as described in Alemdar and Sain (2008). Additionally, one portion of the material was bleached following a conventional Elemental Chlorine Free (ECF) bleaching sequence $\left.\left(\mathrm{D}_{0} \mathrm{EpD}\right)_{1}\right)$, where the first and third steps used sodium chlorite $\left(\mathrm{NaClO}_{2}\right)$ and the 
second step was a hydrogen peroxide $\left(\mathrm{H}_{2} \mathrm{O}_{2}\right)$ supplemented alkaline extraction process utilizing sodium hydroxide $(\mathrm{NaOH})$. Details of the bleaching process are as follows; first, $\mathrm{NaClO}_{2}$ was incorporated into the fibers at $5 \mathrm{wt} \%$ and kept at $70{ }^{\circ} \mathrm{C}$ for 1 hour at a $\mathrm{pH}$ of 2.8. Second, $\mathrm{H}_{2} \mathrm{O}_{2}$ supplemented alkaline extraction was performed on the fibers at $4 \mathrm{wt} . \%$, adding $\mathrm{NaOH}$ until $\mathrm{pH}$ was 10.5 and left for 2 hours at $80{ }^{\circ} \mathrm{C}$. Finally, $\mathrm{NaClO}_{2}$ was added to the fibers at $2 \mathrm{wt} \%$ and maintained at $70{ }^{\circ} \mathrm{C}$ for $1 \mathrm{~h}$ at a $\mathrm{pH}$ of 2.8. Unless clarified in the text, all the concentrations in the text are expressed on a dry mass basis.

For charge density measurements, $0.01 \mathrm{~N}$ polydiallyldimethylammonium chloride (Poly-DADMAC, sample \#920) and 0.01 N polyvinylsulfuric acid potassium salt (PVSK, sample \#919) were purchased from BTG Americas Inc. A low viscosity silicon oil (Brookfield 1000, $980 \mathrm{cp}$ ) purchased from Brookfield Engineering Laboratories, Inc., was used to seal the sample edge for ensuring no solvent evaporation during rheological measurements.

\section{Methods}

\section{CNF production}

Prior to the defibrillation process, suspensions containing $2.0 \mathrm{wt} . \%$ of the fibers mentioned above were prepared using deionized water. The suspensions were then subjected to mechanical defibrillation using a Masuko supermasscolloider MKZA10-15J IV (Masuko Sangyo Co., Ltd., Japan), passing them ten times between one stationary and one rotating stone. The same procedure was performed for both kraft and soybean fibers. After the mechanical treatment, a $\sim 2.0 \mathrm{wt} \%$ cellulose nanofibril with a gel-like consistency was obtained. For this work, the abbreviations BCNF and LCNF correspond to bleached and unbleached cellulose nanofibrils, respectively. Additionally, the prefixes w- and sbhave been utilized to indicate the raw materials wood and soybean, respectively. For example, sb-BCNF represents bleached cellulose nanofibrils made from soybean. The materials studied in this work are sb-BCNF, sb-LCNF, w-BCNF, and w-LCNF.

\section{Characterization of CNF suspensions}

Dry content, $p H$ and surface charge. Dry content of the samples was measured following TAPPI Standard T550 om-08. Measurements were performed by triplicate, and the results were averaged. Equation 1 was utilized for the calculations of moisture content as:

$$
M C \%=\frac{\text { mass }_{\text {wet }}-\text { mass }_{d r y}}{m a s s_{d r y}} \times 100 \%
$$

Then, the dry content was calculated as the difference between $100 \%$ and the MC\% value. The $\mathrm{pH}$ of the CNF suspensions was assessed by using a VWR® SympHony Benchtop Multiparameter Meter B30PCI. Samples were measured with 15 runs, with the average calculated and reported. The charge density of the nanofibers was measured using a Chemtrac Lab Charge Analyzer (Chemtrac Systems Inc., Model LCA01, USA), following a protocol adapted from Carrasco et al. (1998). Negatively charged CNF suspensions were prepared at 0.04 wt.\% consistency in ultrapure water. Samples were placed in an ice bath, where they were sonicated for $10 \mathrm{~min}$ using a Sonics Vibra Cell Sonicator (Sonics \& Materials, Inc., Newton, CA, Model VC750). Subsequently, $25 \mathrm{~mL}$ of $0.01 \mathrm{~N}$ polyDADMAC was mixed with $15 \mathrm{~mL}$ of CNF suspension, and the mixture was centrifuged at $3000 \mathrm{rpm}$ for $15 \mathrm{~min}$ in an Eppendorf Centrifuge 5415R (Eppendorf AG, 
Hamburg). After centrifugation, $10 \mathrm{~mL}$ of the supernatant was analyzed in the charge analyzer using the anionic polymer $0.01 \mathrm{~N}$ PSVK as the titrant. Charge density was measured with 6 runs and calculated using Eq. 2 (Carrasco et al. 1998),

$$
\text { Charge density }=\frac{\left(C * V_{c}-A * V_{A}\right)}{W}
$$

where $C$ is the concentration of the cationic polymer polyDADMAC, $V_{C}$ is the volume of polyDADMAC, $A$ is the concentration of the anionic polymer (PVSK), $V_{A}$ is the volume of PVSK, and $W$ is the weight of CNF that is consumed to reach a streaming current value (SCV) equal to zero.

Chemical composition. The chemical composition of the nanofiber suspensions was analyzed by Fourier-transform infrared spectroscopy with attenuated total reflectance accessory (ATR-FTIR). This was performed using a Perkin Elmer Spotlight 400 FTIR imaging system equipped with deuterated triglycine sulfate DTGS detector and built-in ATR module with a germanium crystal. All spectra were recorded over the spectral range from 4000 to $400 \mathrm{~cm}^{-1}$ at room temperature and after 64 scans.

Thermal behavior. The thermal stability of the samples was assessed by thermogravimetric analysis (TGA) with a TGA-50 from Shimadzu (Kyoto, Japan) using nitrogen as a sweep fluid at a flow rate of $20 \mathrm{~mL} / \mathrm{min}$. The samples were placed in aluminum pans and heated from 10 to $600{ }^{\circ} \mathrm{C}$ at a heating rate of $10^{\circ} \mathrm{C} / \mathrm{min}$. About $15 \mathrm{mg}$ of each sample was analyzed with measurements performed in duplicate. The data was processed with the Shimadzu TA60 software (version 2.11).

Morphology. The morphology of the cellulose nanofibers was investigated by atomic force microscopy (AFM) utilizing an Anton Paar TOSCA ${ }^{\mathrm{TM}} 400$ AFM (Graz, Austria). The images were obtained with a silicon cantilever in tapping mode, and the scan size area was set at $3 \times 3 \mu \mathrm{m}$. Data visualization and analysis was performed with Gwyddion open software (Source Forge, Version 2.49). For AFM imaging, before the CNF deposition, silicon surfaces were cleaned using UV ozone for $30 \mathrm{~min}$ and submerged for 15 min into $0.1 \mathrm{wt} \%$ polyethylenimine (PEI), which was used as anchoring solution. CNF suspensions were prepared at $0.01 \mathrm{wt} . \%$ and placed in a cold bath to avoid sample heating while sonicating using a Vibra Cell sonicator (Newtown, CA) for $10 \mathrm{~min}$ with $20 \mathrm{~kW}$ and $25 \%$ amplitude to promote delamination and prevent their agglomeration. Then, $80 \mu \mathrm{L}$ of suspension was spin coated onto the PEI-silicon at $3200 \mathrm{rpm}$ for $1 \mathrm{~min}$. Surfaces were placed in the oven at $80{ }^{\circ} \mathrm{C}$ for $20 \mathrm{~min}$ and stored in a desiccator until use.

Crystallinity. X-ray powder diffraction (XRD) was performed using a 1-Dimension Bruker AXS D8 Discover equipped with a LYNXE detector and $\mathrm{Cu} \mathrm{K} \alpha$ irradiation. Measurements were performed at a continuous scan speed of $0.1 \mathrm{~second} / \mathrm{step}$, from 5 to 90 degrees. Data was acquired using the DiffracPlus Eva version 13.0.0.3 by Bruker. The crystallinity index (CI) was calculated utilizing Segal's method, defined by Eq. 3 (Segal et al. 1959):

$$
C I=\frac{I_{002}-I_{A m}}{I_{002}}
$$

Rheological behavior. Rheological properties of the prepared bleached and unbleached CNF from soybean and hardwood sources were measured at $25^{\circ} \mathrm{C}$ using an Anton Paar Physica MCR301 (Graz, Austria) strain-controlled rotational rheometer. Prior to the measurements, $2.0 \mathrm{wt} \%$ dispersions were tip-sonicated, and then allowed $3 \mathrm{~h}$ of 
relaxation before loading onto the rheometer. Rheological measurements were primarily performed on $25 \mathrm{~mm}$ diameter parallel plates. Some tests were repeated using other geometries to ensure that there were no artifacts in the data. All tests were performed with a silicon oil coating along the edge of the fixture and a solvent trap of deionized water to prevent water loss during testing. After loading the sample on the rheometer, the sample was allowed to equilibrate for $10 \mathrm{~min}$ before investigation of rheological properties. A preliminary shear protocol using a shear rate of $0.001 \mathrm{~s}^{-1}$ for $20 \mathrm{~min}$ was used to further reduce artifacts from shear induced microstructural changes during sample loading. The dispersion microstructures were investigated with oscillatory shear measurements, where amplitude sweeps were used to determine the linear viscoelastic region (LVR). Then, frequency sweeps were performed at $0.2 \%$ strain (within the LVR) to measure the storage $\left(G^{\prime}\right)$ and loss $\left(G^{\prime \prime}\right)$ moduli as a function of angular frequency $\omega$. Steady shear viscosity tests were then performed to investigate the effect of shear on the structures. Constant shear step rate tests were performed to determine the time for samples to reach a steady viscosity, which was then used for the flow curves. Flow curves were performed to determine the dependence of steady shear viscosity $\eta$ on shear rate $\dot{\gamma}$.

\section{RESULTS AND DISCUSSION}

\section{Characterization of CNFs Suspensions}

Lignin content, dry content, $\mathrm{pH}$, and surface charge

The main components of soybean hulls are cellulose $(39.7 \%)$, hemicellulose $(25.5 \%)$, pectin (12.7\%), lignin (9.1\%), proteins (13.1\%), and ash (0.6\%) (Cassales et al. 2011). Nevertheless, after chemical treatment of the hulls to obtain fibers, these components are partially eliminated, while only cellulose, hemicelluloses, pectin, and lignin remain (Alemdar and Sain 2008). For wood as a raw material, the main components for hardwood samples are cellulose $(41.0 \%)$, hemicellulose $(29.8 \%)$, lignin $(22.0 \%)$, and extractives (3.2\%) (Sjostrom et al. 1993). Like soybean hulls, after the pulping process of wood to obtain cellulose fibers, the chemical composition changes due to the partial elimination of some components (Smook 2016). It is worth mentioning that the differences in the chemical composition of the starting materials will be translated to differences in the nanocelluloses fibers made thereof. Table 1 shows the lignin content, dry content, $\mathrm{pH}$, and charge density data for the set of samples utilized in this work.

As shown in Table 1, sb-LCNF showed the highest value of charge density. To corroborate the differences of the mean between the samples, a statistical analysis with ANOVA was performed. By performing a Tukey test, the charge density for sample sbLCNF was confirmed to be significantly different from the rest of the specimens. However, the other three were not significantly different. The high charge density on the sb-LCNF could be attributed to the presence of pectin in the sample. Furthermore, the charge density of the wood CNFs was smaller than sb-LCNF and slightly greater than sb-BCNF. These discrepancies may be attributed not only to the presence of pectin in the sb-LCNF sample but also to the effect of the chemical treatments on the surface of the wood fibers. It is known that during kraft pulping, free phenolic hydroxyl groups are formed, together with lignin-carbohydrate complexes (Iglesias et al. 2020a), which modify the properties of the fibers. 
Table 1. Lignin Content, Dry Content, $\mathrm{pH}$, and Charge Density

\begin{tabular}{|l|c|c|c|c|c|}
\hline Property & Unit & w-BCNF & w-LCNF & sb-BCNF & sb-LCNF \\
\hline Lignin content & $\%$ & $<^{*}$ & $2.3^{*}$ & $<1^{* *}$ & $\sim 2.5^{\star *}$ \\
\hline Dry Content & wt. $\%$ & $1.9 \pm 0.0$ & $2.0 \pm 0.0$ & $2.0 \pm 0.0$ & $2.1 \pm 0.0$ \\
\hline pH & - & $6.1 \pm 0.0$ & $6.1 \pm 0.0$ & $6.0 \pm 0.0$ & $6.0 \pm 0.0$ \\
\hline Charge density & $\mu \mathrm{eq} / \mathrm{gr}$ & $206.1 \pm 10.0$ & $206.7 \pm 6.0$ & $191.1 \pm 43.5$ & $310.0 \pm 24.1$ \\
\hline
\end{tabular}

*Lignin content was estimated from the Kappa number provided in the datasheet.

**Lignin content was estimated from Alemdar and Sain 2008.

\section{Thermal behavior}

The thermal behavior of the samples is presented in Fig. 1 . The corresponding $T_{\text {onset }}$ and $T_{\max }$ values of all the samples are summarized in Table 2, where $T_{\text {onset }}$ refers to the initial visually apparent inflection mass decrease and $T_{\max }$ refers to the temperature corresponding to the maximum in the derivative peak. Analysis of the graphs shows that $T_{\text {onset }}$ is around $346{ }^{\circ} \mathrm{C}$ for wood samples, while for soybean samples this temperature increases to approximately $361{ }^{\circ} \mathrm{C}$. The derivative peaks convey that the values of $T_{\max }$ were equivalent; the actual values of $406.5^{\circ} \mathrm{C}$ and $408.5^{\circ} \mathrm{C}$ are within the instrument error. Cellulose and hemicelluloses present degradation temperature ranges between $315-400{ }^{\circ} \mathrm{C}$ and $220-315{ }^{\circ} \mathrm{C}$, respectively (Yang et al. 2007). This explains the peak breadth for all four samples. Regarding the wood nanofibers, the w-LCNF mass loss derivative curve shows an additional small peak between 200 and $250{ }^{\circ} \mathrm{C}$. This is attributed to lignin degradation; it has been reported that due to its complex composition, lignin decays in a range of temperatures from 200 to over $500{ }^{\circ} \mathrm{C}$ (Brebu and Vasile 2010). Alemdar and Sain (2008), reported that untreated soybean hull fibers start to decompose at $209^{\circ} \mathrm{C}$, while the nanofibers produced from those fibers have a notably higher decomposition temperature of $290{ }^{\circ} \mathrm{C}$.

Table 2. $T_{\text {onset }}$ and $T_{\max }$ Temperatures for Wood and Soybean CNFs

\begin{tabular}{|l|c|c|c|c|}
\hline Sample & w-BCNF & w-LCNF & sb-BCNF & sb-LCNF \\
\hline $\boldsymbol{T}_{\text {onset }}\left({ }^{\circ} \mathbf{C}\right)$ & 346.0 & 346.8 & 361.0 & 361.0 \\
\hline $\boldsymbol{T}_{\max }\left({ }^{\circ} \mathbf{C}\right)$ & 406.5 & 406.5 & 408.5 & 408.5 \\
\hline
\end{tabular}

In the set of nanocellulose samples, greater thermal stability was observed for soybean CNFs compared with wood CNFs. These results are in accordance with the data reported by Ferrer et al. (2016), where the decomposition temperature for soybean CNF was $305{ }^{\circ} \mathrm{C}$ while for bleached CNF from wood, it occurred at $282{ }^{\circ} \mathrm{C}$. The amount of hemicelluloses in hardwood CNFs with similar characteristics to those presented in this work, has been reported to be between $19.2 \%$ and $18.4 \%$ (Iglesias et al. 2020b). In contrast, utilizing the extraction method proposed by Alemdar and Sain (2008), the CNFs from soybean hulls contained only $3.5 \%$ of hemicelluloses after the alkali and acidic treatment. As a result, the lower thermal stability for wood CNFs can be attributed to the higher amount of hemicelluloses in these samples that have a low degradation temperature compared to the other components. 

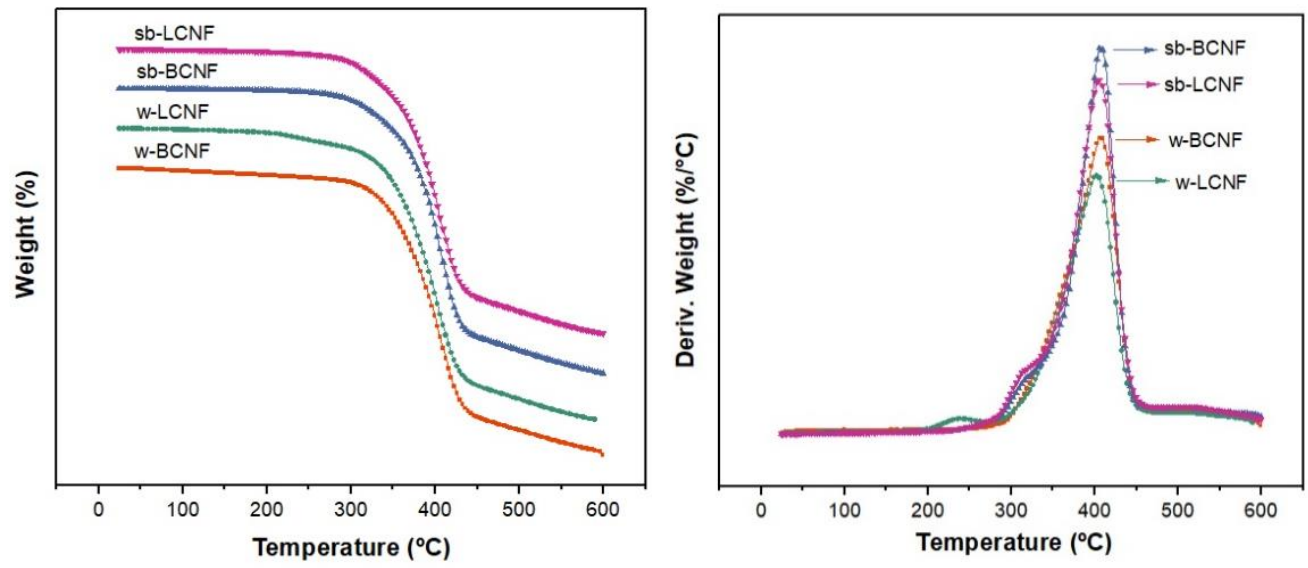

Fig. 1. TGA curves in nitrogen atmosphere including weight $(\%)$ and the derivative weight $(\% / 0 \mathrm{C})$ of the set of nanocellulose samples as indicated in the plots

\section{Chemical composition}

As can be observed in Fig. 2 in the region between 4000 and $3250 \mathrm{~cm}^{-1}$, the FTIR spectra are dominated by the peaks at $3335 \mathrm{~cm}^{-1}$ reflecting the aromatic and aliphatic stretching vibrations for $\mathrm{O}-\mathrm{H}$ groups, which is consistent with spectra previously reported (Alemdar and Sain 2008; Tang et al. 2020a). Additionally, in the range between 2922 and $2855 \mathrm{~cm}^{-1}$, the bands correspond to stretching vibrations of $\mathrm{CH}_{3}, \mathrm{CH}_{2}$, and $\mathrm{CH}$ (Debiagi et al. 2020).

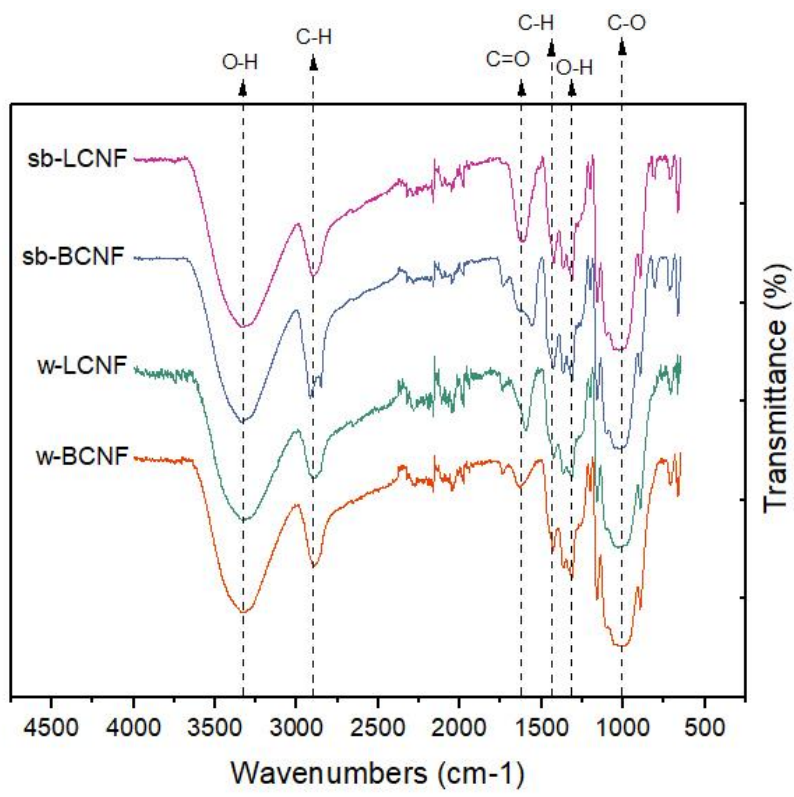

Fig. 2. FTIR spectra of samples (from top to bottom) sb-LCNF, sb-BCNF, w-BCNF, and w-LCNF

At $1604 \mathrm{~cm}^{-1}$ there is a peak for the sb-LCNF sample, which is assigned to $\mathrm{C}=\mathrm{O}$ stretching vibrations corresponding to carboxylic groups. As described by Alemdar and Sain (2008), this peak can be related to the absorption of water. Its intensity decreases for sb-BCNF due to the partial removal of hemicelluloses during the bleaching process. The differences in relative peak intensities among the different samples can be attributed to the different initial chemical compositions of the raw material and the strength of the chemical 
treatment utilized to obtain the nanofibers. The absorption peaks at 1430 and $1319 \mathrm{~cm}^{-1}$ result from $\mathrm{CH}_{2}$ and $\mathrm{O}-\mathrm{H}$ deformations, respectively (Larkin 2011). Additionally, the shoulder at $1240 \mathrm{~cm}^{-1}$ corresponds to bending vibrations from $\mathrm{O}-\mathrm{H}, \mathrm{C}-\mathrm{H}$, and $\mathrm{CH}_{2}$. Finally, the high-intensity peak at $1026 \mathrm{~cm}^{-1}$ observed in all samples is attributed to $\mathrm{C}-\mathrm{O}$ stretching vibrations from polysaccharides and lignin, confirming the presence of the latter on the samples.

\section{Crystallinity}

X-ray powder diffraction (XRD) was used to measure the crystallinity of nanocellulose samples. Crystallinity is affected by both the crystallinity of the precursor material and the chemical treatment utilized to produce the samples (Ferrer et al. 2016). All the samples exhibited a major peak at a $2 \theta$ value between $21.5^{\circ}$ and $22.5^{\circ}$, and a smaller peak around $15.5^{\circ}$. Data were normalized and presented in Fig. 3. Results showed that w$\mathrm{BCNF}$ and $\mathrm{w}-\mathrm{LCNF}$ had a CI of $40.42 \pm 0.01 \%$ and $46.14 \pm 0.03 \%$, respectively. Additionally, the CI for soybean samples was $54.07 \pm 0.01 \%$ and $48.09 \pm 0.01 \%$ for sb$\mathrm{BCNF}$ and sb-LCNF, respectively. Based on the obtained data, soybean CNFs had a higher crystallinity than wood CNFs. The present results are in agreement with those reported by Ferrer et al. (2016). Comparing the CNFs from both sources, the differences in crystallinity for soybean and wood nanofibrils could be also attributed to the preparation process, which may produce heavier damage to the supramolecular structure of the wood fibers, thus decreasing their crystallinity. This can be correlated in the following section with the morphology of the samples.

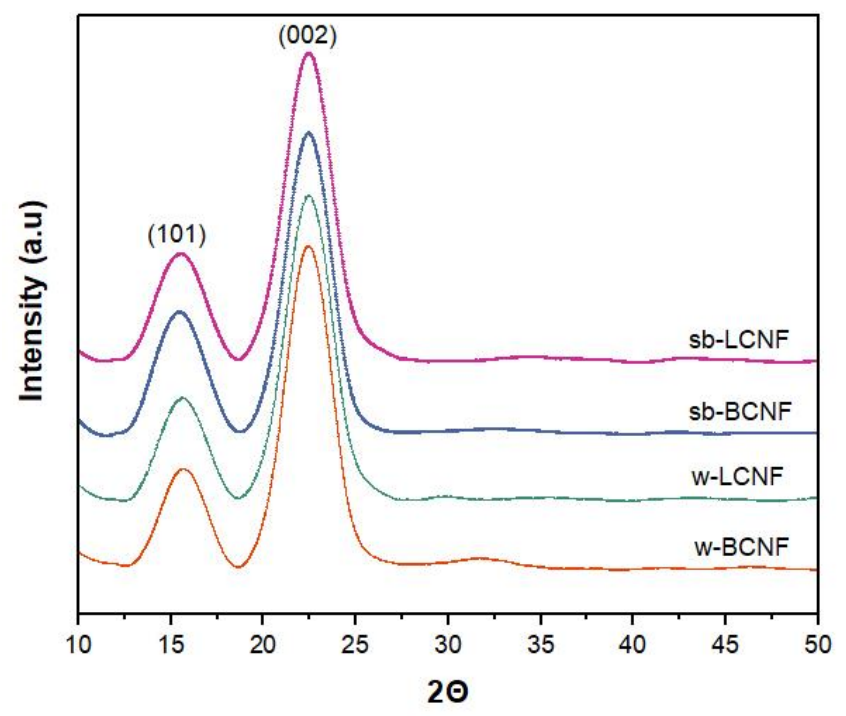

Fig. 3. XRD spectra of samples (from bottom to top) w-BCNF, w-LCNF, sb-BCNF, and sb-LCNF

Additionally, the present crystallinity results were in accordance with the findings obtained in the thermal analysis discussed in the previous sections, where larger degradation temperatures can be related to larger crystallinity of the samples due to the increase in cellulose content (Espinosa et al. 2017; Debiagi et al. 2020; Yuan et al. 2021).

Regarding the soybean CNFs, the higher CI of sample sb-BCNF compared with sbLCNF is related to the reduction of lignin, hemicelluloses, and pectin content, which are 
the components that contribute to the amorphous or non-crystalline material (Espinosa et al. 2017). With regards to the wood samples, the w-BCNF shows a lower crystallinity than w-LCNF, even when the latter contains lignin and hemicelluloses. A disadvantage of the chemical process necessary to bleach the wood sample is the possibility of degrading and damaging the samples, which reduces their crystallinity (Debiagi et al. 2020).

\section{Morphology}

The morphology of the samples was analyzed by AFM, and the topographic images are presented in Fig. 4. CNF from wood had a greater amount of fibril bundles when compared with CNF from soybean hulls. The AFM images suggest heterogeneous samples, containing both thick and long microfibril and microparticle bundles as well as a network of very fine irregular fibrils. CNFs from soybean hulls seem to be thicker and shorter than the CNFs from wood. Nevertheless, a broader understanding of the morphology of the samples will be discussed in depth in the rheological behavior of the suspensions. As mentioned in the previous section, the morphology of the samples could be also related to their crystallinity. CNFs from soybean hulls present thicker structures than CNFs from wood. It is possible that during the production process, soybean fibers suffered less damage than wood fibers, which could be the reason for the higher crystallinity of sb-CNFs.
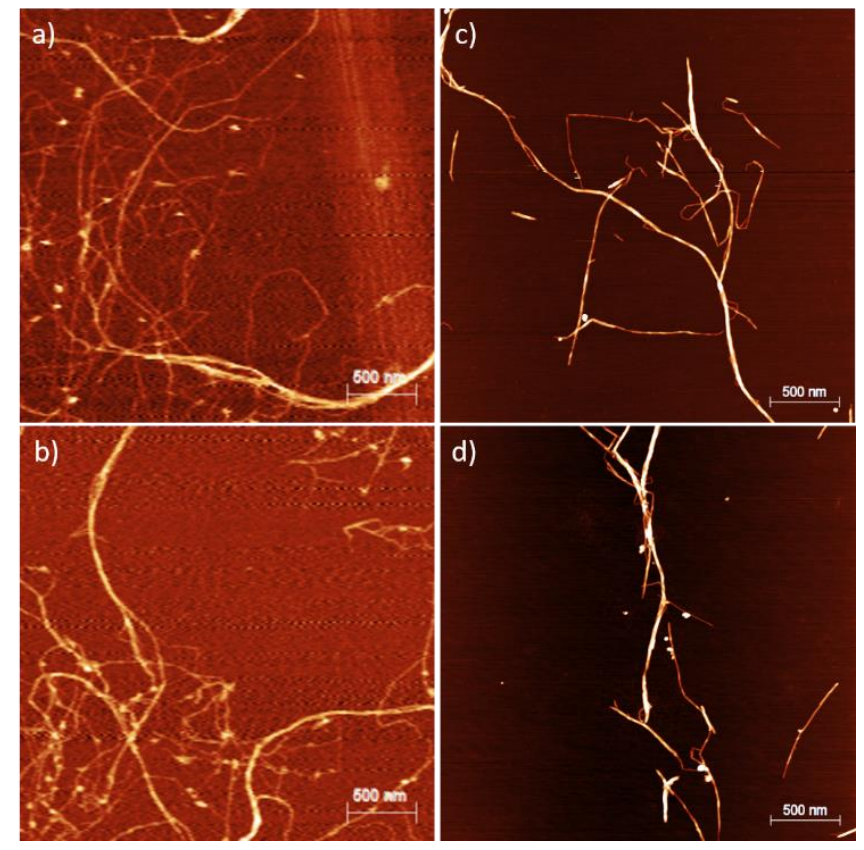

Fig. 4. AFM topographic microscopies, of a) w-BCNF, b) w-LCNF, c) sb-BCNF, and d) sb-LCNF. With image size of $3 \mu \mathrm{m} \times 3 \mu \mathrm{m}$ and scale bar of $500 \mathrm{~nm}$

\section{Rheological behavior}

The rheological behavior of dispersions of all wood and soybean CNFs dispersions was determined. Rheology is a bulk measurement method that is sensitive to solid content, charge density, $\mathrm{pH}$, and morphology (Macosko 1994). The CNFs from wood showed a viscosity ten times larger than that corresponding to soybean CNFs. The sb-LCNF had the lowest shear viscosity value. The reduction in viscosity could be attributed to the interparticle repulsion, which favors the colloidal stability of the sample, decreasing the 
tendency of the particles to form agglomerates (Hubbe et al. 2017b). Additionally, the presence of hemicelluloses and pectin include additional negative charges to the suspensions which may contribute to the repulsion between fibers (Hubbe et al. 2008). Nevertheless, charge density is not the only factor affecting viscosity. Although sb-BCNF shows a slightly lower charge density than the CNFs from wood, the sb-LCNF has a lower viscosity than those samples. This variance could be attributed to the differences in morphology; while wood samples are entangled and long structures, soybean CNFs are short fibers that can be expected to have higher mobility and therefore, lower viscosity.

As expected, all the samples exhibited non-Newtonian shear-thinning behavior (Fig. 5), such that the viscosity of the samples decreased as a function of shear rate. (Pääkko et al. 2007; Iotti et al. 2011; Iglesias et al. 2020b). This is attributed to alignment of the fibrils with increasing shear rate. The steady shear rheology data was fit to models to enable more detailed comparison between samples. The data is presented in Table 3 and the power index < 1 confirmed the shear-thinning behavior for non-Newtonian fluids (Macosko 1994). Additionally, the power law index is slightly larger for soybean samples than for the wood samples, which could indicate a dependence on the raw material of the CNFs.

Table 3. Power-law and Herschel-Bulkley Parameters Fitted to the Data $\eta=k \dot{\gamma}^{n-1}$ for sb-BCNF, w-BCNF, and w-LCNF, and $\tau=\tau_{0}+k \dot{\gamma}^{n}$ for Sample sb-LCNF.

\begin{tabular}{|l|c|c|c|}
\hline Sample & $\boldsymbol{k}$ & $\boldsymbol{n}$ & $\boldsymbol{\tau}_{\mathbf{0}} \mathbf{( P a )}$ \\
\hline sb-BCNF & 53.7 & 0.13 & - \\
\hline sb-LCNF & 4.3 & 0.60 & 14.0 \\
\hline w-BCNF & 97.8 & 0.06 & - \\
\hline w-LCNF & 256.9 & 0.08 & - \\
\hline
\end{tabular}

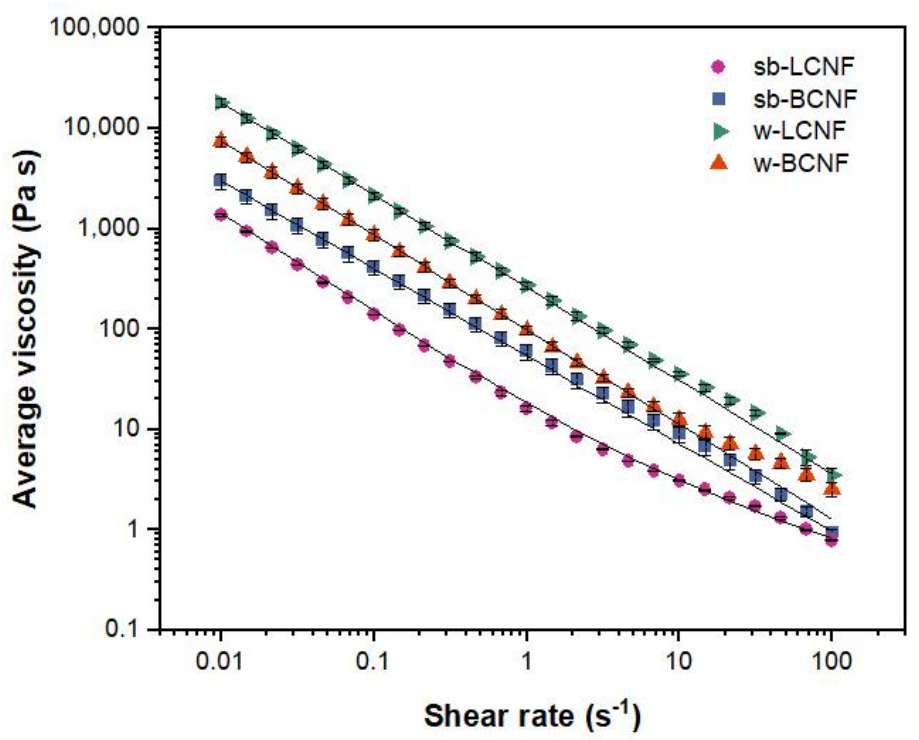

Fig. 5. Steady state flow curves for $\operatorname{sb-LCNF}(\bullet), \operatorname{sb}-\operatorname{BCNF}(\bullet), w-\operatorname{LCNF}(\bullet)$, and $w-\operatorname{BCNF}(\boldsymbol{\Delta})$. The curves represent model fits to the data, where the power-law model was fit to sb-BCNF, $\mathbf{w}-$ LCNF, and w-BCNF and the Herschel-Bulkley model was fit to sb-LCNF. 
For the w-BCNF, w-LCNF, and sb-BCNF, the power-law model (Eq. 4) described the behavior.

$$
\eta=k \dot{\gamma}^{n-1}
$$

where $\eta$ is the viscosity, $k$ is the consistency index, $\dot{\gamma}$ is the shear rate, and $n$ is the power law index. However, sample sb-LCNF exhibited a well-defined yield stress. There was one order of magnitude decrease in viscosity at a shear stress of $14 \mathrm{~Pa}$. As a result, the HerschelBulkley model (Eq. 5) provided a better fit for the sb-LCNF data,

$$
\begin{aligned}
& \tau=\tau_{0}+k \dot{\gamma}^{n} \\
& \eta=\tau * \dot{\gamma}
\end{aligned}
$$

where $\tau$ is the shear stress at a given shear rate and $\tau_{0}$ is the yield stress. This result is surprising, since it was the only sample to show a yield stress, but in other studies specimens with the highest charge density show decreased yield stress due to repulsion between the fibers (Horvath and Lindström 2007). However, in this study both the differences in the morphology (Fig. 4) and chemistry affected the flow behavior. As mentioned previously, soybean hulls contain pectin, interacting in the primary cell wall with cellulose and hemicelluloses (Medic et al. 2014). Although the chemical treatment reduces the amount of hemicelluloses, lignin, and pectin on the extracted soybean fibers (Alemdar and Sain 2008), remnants of pectin on the sample could act as a gelling agent (Monsoor and Proctor 2001) between the different lignocellulosic components. This could restrict the movement of the fibers and generate the yield stress observed on the unbleached soybean CNF.

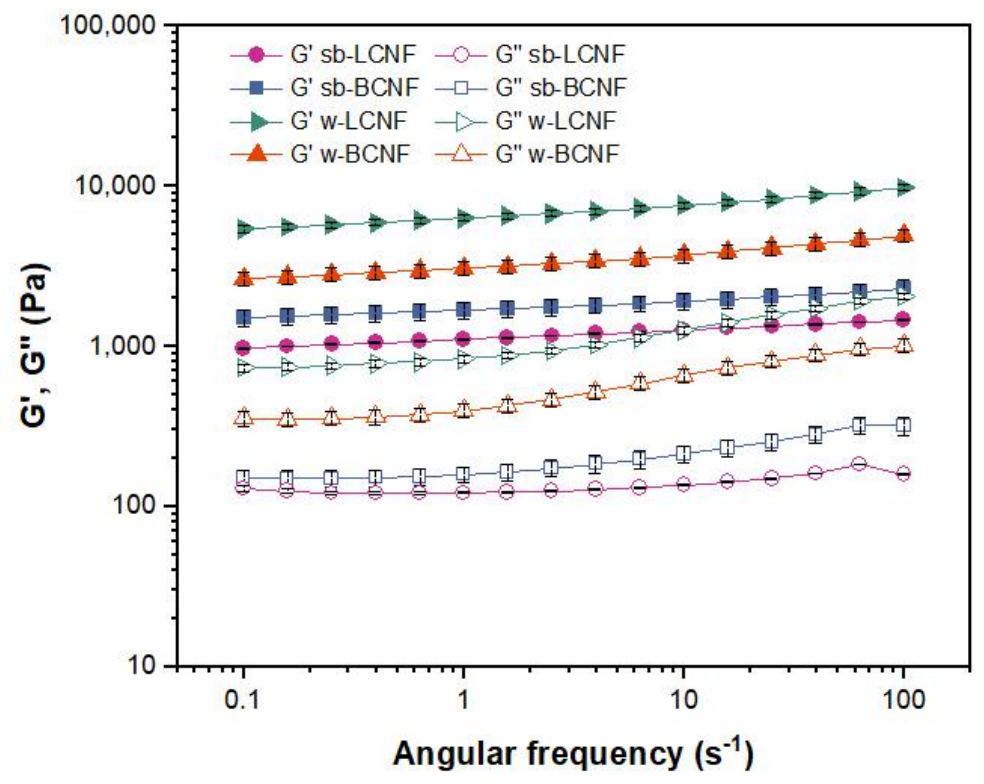

Fig. 6. Oscillatory frequency sweeps for the set of nanocelluloses at $0.2 \%$ of strain. G' (bold) and G" (empty) for sb-LCNF $(\bullet, \circ)$, $\operatorname{sb-BCNF}(\bullet, \square), \operatorname{w-LCNF}(\triangleright, \triangleright)$, and w-BCNF $(\boldsymbol{\Delta}, \triangle)$

Small amplitude oscillatory shear (SAOS) was used to gain more insight into the samples' viscoelastic nature. Figure 6 shows that all samples were primarily elastic, the 
storage moduli were greater than the loss moduli $\left(G^{\prime}>\underline{G}^{\prime \prime}\right)$ with $\tan (\delta)=G^{\prime \prime} / G^{\prime} \sim 0.1$. For both $G$ ' and $G$ ' the wood samples had higher values than the soybean CNFs. In a similar fashion as the steady shear results, this can be attributed to the more entangled structures observed in the AFM images. Compared to the soybean CNFs, wood CNFs show higher $G$ ' and $G$ ', which could be related to the thick and long fibers bundles observed in the AFM images. Interestingly, the lower slope of the soybean samples suggests that they were the closest to achieving percolated network formation.

\section{CONCLUSIONS}

Cellulose nanofibrils (CNFs) were prepared from two different sources, wood and soybean hulls. In order to better understand how the chemical composition of each raw material affects the characteristics of nanocellulose suspensions, CNF was prepared from bleached and unbleached fibers from each of the materials.

The samples were fully characterized in terms of chemical composition, morphology, thermal, and rheological behavior. A higher thermal stability of the soybean hulls samples was observed compared with the CNFs produced from wood. This was correlated with a higher crystallinity of the soybean hulls nanocellulose samples.

Additionally, the morphology of the samples showed entangled structures for wood samples, which was in agreement with the higher viscosity and more elastic behavior observed in the rheological assessment. Furthermore, the charge density of these samples fell between those obtained for sb-LCNF and sb-BCNF. Although no trend was observed between wood and soybean fibers in terms of charge density, it is concluded that the differences are not only due to their chemical composition but also due to the chemical treatments used to produced them.

Finally, the samples were fully characterized in terms of their rheological behavior. All the specimens presented a shear-thinning behavior and were primarily elastic. This was more noticeable for wood CNFs, which was mainly attributed to the morphology of these fibers. Furthermore, the modeling of the rheological properties and the yield stress found on the sb-LCNF when performing oscillatory measurements also introduced a novelty on the rheological behavior of the samples.

\section{ACKNOWLEDGMENTS}

The authors would like to thank Dr. Orlando Rojas (Aalto University) for granting the access to AFM facilities, Dr. Beatriz Vega for assisting with the AFM measurements, and Dr. Charles Frazier and Shannon Lenahan (Virginia Tech University) for assisting with the XRD measurements. This work was supported by the USDA National Institute of Food and Agriculture, Hatch program (ALA013-17003) and McIntire-Stennis program (1022526). The School of Forestry and Wildlife Sciences at Auburn University's financial support to complete this work is much appreciated. 


\section{REFERENCES CITED}

Ahuja, D., Kaushik, A., and Singh, M. (2018). "Simultaneous extraction of lignin and cellulose nanofibrils from waste jute bags using one pot pre-treatment," Int. J. Biol. Macromol. DOI: 10.1016/j.ijbiomac.2017.09.107

Aksoy, B., Jiang, Z., Aksoy, M., and Beck, B. (2020). "Using liberated and modified fibers, micro, nano fibers and cellulose from lignocellulosic plant fibers, and algae as feed binders in compound feeds," US Provisional patent application No.: 63/054,907.

Alemdar, A., and Sain, M. (2008). "Biocomposites from wheat straw nanofibers: Morphology, thermal and mechanical properties," Compos. Sci. Technol. 68, 557565. DOI: 10.1016/j.compscitech.2007.05.044

Brebu, M., and Vasile, C. (2010). "Thermal degradation of lignin-A review," Cellul. Chem. Technol. 44, 353-363.

Carrasco, F., Mutjé, P., and Pelach, M. A. (1998). "Control of retention in paper-making titration and zeta potential techniques," Wood Sci. Technol. 32, 145-155. DOI: 10.1007/BF00702595

Cassales, A., de Souza-Cruz, P. B., Rech, R., and Záchia Ayub, M. A. (2011). "Optimization of soybean hull acid hydrolysis and its characterization as a potential substrate for bioprocessing," Biomass and Bioenergy 35(11), 4675-4683. DOI: 10.1016/j.biombioe.2011.09.021

Chen, Y., Wu, Q., Huang, B., Huang, M.-J., and Ai, X.-L. (2015). "Isolation and characteristics of cellulose and nanocellulose from lotus leaf stalk agro-wastes," BioResources 10(1), 684-696. DOI: 10.15376/biores.10.1.684-696

Debiagi, F., Faria-Tischer, P. C. S., and Mali, S. (2020). "Nanofibrillated cellulose obtained from soybean hull using simple and eco-friendly processes based on reactive extrusion," Cellulose 72(4), 1975-1988. DOI: 10.1007/s10570-019-02893-0

Deepa, B., Abraham, E., Cordeiro, N., Mozetic, M., Mathew, A. P., Oksman, K., Faria, M., Thomas, S., and Pothan, L. A. (2015). "Utilization of various lignocellulosic biomass for the production of nanocellulose: A comparative study," Cellulose 22, 1075-1090. DOI: $10.1007 / \mathrm{s} 10570-015-0554-\mathrm{x}$

Espinosa, E., Domínguez-Robles, J., Sánchez, R., et al. (2017). "The effect of pretreatment on the production of lignocellulosic nanofibers and their application as a reinforcing agent in paper," Cellulose 24, 2605-2618. DOI: 10.1007/s 10570-0171281-2

FAOSTAT (2019). http://www.fao.org/faostat/en/\#data/QC

Feng, Y. H., Cheng, T. Y., Yang, W. G., Ma, P.-T., He, H.-Z., Yin, X.-C., and Yu, X.-X. (2018). "Characteristics and environmentally friendly extraction of cellulose nanofibrils from sugarcane bagasse," Ind. Crops Prod. 111, 285-291. DOI: 10.1016/j.indcrop.2017.10.041

Ferrer, A., Salas, C., and Rojas, O. J. (2016). "Physical, thermal, chemical and rheological characterization of cellulosic microfibrils and microparticles produced from soybean hulls," Ind. Crops Prod. 84, 337-343. DOI:

10.1016/j.indcrop.2016.02.014

Horvath, A. E., and Lindström, T. (2007). "The influence of colloidal interactions on fiber network strength," J. Colloid Interface Sci. 22, 824-830. DOI: 10.1016/j.jcis.2006.08.066

Hubbe, M. A., Ferrer, A., Tyagi, P., Yin, Y., Salas, C., Pal, L., and Rojas, O. J. (2017a). 
"Nanocellulose in thin films, coatings, and plies for packaging applications: A review," BioResources 12, 2143-2233.

Hubbe, M. A., Tayeb, P., Joyce, M., Tyagi, P., Kehoe, M., Dimic-Misic, K., and Pal, L. (2017b). "Rheology of nanocellulose-rich aqueous suspensions: A review,"

BioResources 12(4), 9556-9661. DOI: 10.15376/biores.12.1.2143-2233

Hubbe, M. A., Rojas, O. J, Lucia, L, and Sain, M. (2008). "Cellulosic nanocomposites: A review," BioResources 3, 929-980. DOI: 10.15376/biores.3.3.929-980

Hult, E. L., Iotti, M., and Lenes, M. (2010). "Efficient approach to high barrier packaging using microfibrillar cellulose and shellac," Cellulose 17, 575-586. DOI: 10.1007/s 10570-010-9408-8

Iglesias, M. C., Gomez-Maldonado, D., Via, B. K., Jiang, Z.-H., and Peresin, M. S. (2020a). "Pulping processes and their effects on cellulose fibers and nanofibrillated cellulose properties: A review," For. Prod. J. 70, 10-21. DOI: 10.13073/FPJ-D-1900038

Iglesias, M. C., Shivyari, N., Norris, A., Martin-Sampedro, R., Eugenio, M. E., Lahtinen, P., Auad, M. L., Elder, T., Jiang, Z.-H., Frazier, C. E., and Peresin, M. S. (2020b). "The effect of residual lignin on the rheological properties of cellulose nanofibril suspensions," J. Wood Chem. Technol. 40(6), 370-381. DOI: $10.1080 / 02773813.2020 .1828472$

Iotti, M., Gregersen, Ø. W., Moe, S., and Lenes, M. (2011). "Rheological studies of microfibrillar cellulose water dispersions," J. Polym. Environ. 19, 137-145. DOI: 10.1007/s10924-010-0248-2

Kawamura, S. (1967). "Quantitative paper chromatography of sugars of the cotyledon, hull, and hypocotyl of soybeans of selected varieties," Kagawa University, Departmental Bulletin Paper, 18(2), 117-131.

Klemm, D., Kramer, F., Moritz, S., Lindström, T., Ankerfors, M., Gray, D., and Dorris, A. (2011). "Nanocelluloses: A new family of nature-based materials," Angew. Chemie - Int. Ed. 50, 5438-5466. DOI: 10.1002/anie.201001273

Larkin, P. J. (2011). "Unknown IR and Raman spectra," in: IR and Raman Spectroscopy Principles and Spectral Interpretation, Elsevier, United States, pp. 177-212.

Li, P., Wang, Y., Hou, Q., Liu, H., Liang, C., and Li, X. (2019). "Effect of pretreatment on the structure and properties of nanofibrillated cellulose from soybean residues," BioResources 14(1), 554-560. DOI: 10.15376/biores.14.1.554-560

Macosko, C. W. (1994). "Rheology: Principle, measurements, and applications," WILEY-VCH Verlag GmbH \&Co., Canada

Medic, J., Atkinson, C., and Hurburgh, C. R. (2014). "Current knowledge in soybean composition," J. Am. Oil Chem. Soc. 91, 363-384. DOI 10.1007/s11746-013-2407-9.

Monsoor, M. A., and Proctor, A. (2001). "Preparation and functional properties of soy hull pectin," J. Am. Oil Chem. Soc. 78, article no. 709. DOI: 10.1007/s11746-0010330-z

Pääkko, M., Ankerfors, M., Kosonen, H., Nykänen, A., Ahola, S., Österberg, M., Ruokolainen, J., Laine, J., Larsson, P T., Ikkala, O., and Lindström, T. (2007). "Enzymatic hydrolysis combined with mechanical shearing and high-pressure homogenization for nanoscale cellulose fibrils and strong gels," Biomacromolecules 8, 1934-1941. DOI: 10.1021/bm061215p

Palacios Hinestroza, H., Hernández Diaz, J. A., Esquivel Alfaro, M., Toriz, G., Rojas, O. J., and Sulbarán-Rangel, B. C. (2019). "Isolation and characterization of nanofibrillar cellulose from Agave tequilana Weber bagasse," Adv. Mater. Sci. Eng. 
2019, article no. 1342547. DOI: 10.1155/2019/1342547

Sangeetha, V. H., Varghese, T. O., and Nayak, S. K. (2019). "Isolation and characterisation of nanofibrillated cellulose from waste cotton: Effects on thermomechanical properties of polylactic acid/MA-g-SEBS blends," Iran Polym. J. (English Ed.) DOI: 10.1007/s13726-019-00733-3

Segal, L., Creely, J. J., Martin, A. E., and Conrad, C. M. (1959). "An empirical method for estimating the degree of crystallinity of native cellulose using the X-ray diffractometer," Text. Res. J. 29, 786-794. DOI: 10.1177/004051755902901003

Sjöström, E. (1993). Wood Chemistry, Fundamentals and Applications, Second Ed., Elsevier, Academic Press Inc., California, San Diego.

Smook, G. (2016). "Kraft pulping," in: Handbook for Pulp \& Paper Technologist, $4^{\text {th }}$ Ed., M. Kocurek (ed.), TAPPI Press, Atlanta, pp. 76-85.

Tang, F., Li, S., Yu, H.-Y., Wang, C., Li, Y.-Z., Li, Z.-H., Yao, J.-M., Tang, J.-H., and Zhu, J.-Y. (2020a). "Tailoring commercial cellulose membranes into janus conductive electronic skin via diffusion-controlled polymerization," ACS Sustain. Chem. Eng. 8, 17458-17465. DOI: 10.1021/acssuschemeng.0c05913

Tang, F., Yu, H., Hussain Abdalkarim, S. Y., Sun, J.-H., Fan, X-M., Li, H.-Z., Zhou, Y., and Tam, K. C. (2020b). "Green acid-free hydrolysis of wasted pomelo peel to produce carboxylated cellulose nanofibers with super absorption/flocculation ability for environmental remediation materials," Chem. Eng. J. 395, 125070. DOI: 10.1016/j.cej.2020.125070

Tarrés, Q., Espinosa, E., Domínguez-Robles, J., Rodríguez, A., Mutjé, P., and DelgadoAguilar, M. (2017). "The suitability of banana leaf residue as raw material for the production of high lignin content micro/nano fibers: From residue to value-added products," Ind. Crops Prod. 99, 27-33. DOI: 10.1016/j.indcrop.2017.01.021

USDA (2019). https://quickstats.nass.usda.gov/results/F248CF7B-0665-3904-A2BEF9CF1A1A80DD

Xu, J., Krietemeyer, E. F., Boddu, V. M., Liu, S. X., and Liu, W.-C. (2018). "Production and characterization of cellulose nanofibril (CNF) from agricultural waste corn stover," Carbohydr. Polym. 192, 202-207. DOI: 10.1016/j.carbpol.2018.03.017

Yang, H., Yan, R., Chen, H., Lee, D. H., and Zheng, C.-G. (2007), "Characteristics of hemicellulose, cellulose and lignin pyrolysis," Fuel 86, 1781-1788. DOI: 10.1016/j.fuel.2006.12.013

Yuan, T., Zeng, J., Wang, B., Cheng, Z., and Chen, K.-F. (2021). "Lignin containing cellulose nanofibers (LCNFs): Lignin content-morphology-rheology relationships," Carbohydr. Polym. 254, article no. 117441. DOI: 10.1016/j.carbpol.2020.117441

Article submitted: March 11, 2021; Peer review completed: April 11, 2021; Revised version received and accepted: May 7, 2021; Published: May 13, 2021.

DOI: 10.15376/biores.16.3.4831-4845 